RESEARCH PAPER RP812

Part of Journal of Research of the National Bureau of Standards, Volume 15, July 1935

\title{
INFRARED ARC SPECTRUM OF CHROMIUM
}

\author{
By C. C. Kiess
}

ABSTRACT

The new types of plates, sensitive to infrared light, have made it possible to photograph the arc spectrum of chromium out to 11610 A. Approximately 200 wave lengths have been recorded for this region. Most of these are classified as combinations between terms already known and the new terms, mostly of the triplet system, which are given in the paper.

\section{INTRODUCTION}

Fifteen years ago a survey of the are spectrum of chromium, extending from $5500 \mathrm{~A}$ to the infrared limit photographically attainable at that time, was made in the spectroscopic laboratory of the National Bureau of Standards. ${ }^{1}$ This work not only bridged the gap that had previously existed between the longest known wave length in the red and the beginning of Randall and Barker's ${ }^{2}$ list of infrared lines, observed radiometrically; but also pointed out the first regularities to be noted for this spectrum. An outstanding feature of the chromium are spectrum is the presence of several intense triplets of which some lie in proximity to the boundaries between the traditional actinic, visible, and infrared regions. Thus, for example, the infrared chromium spectrum may be considered as that portion composed of wave lengths greater than those of the strong triplet at 7356 , 7400 , and $7462 \mathrm{~A}$. The production of new types of photographic plates in recent years by the Eastman Kodak Co., far surpassing in sensitivity those used in this earlier work, has made it desirable to resurvey the infrared region of the chromium arc spectrum, especially since our present knowledge of its term structure enables us to predict lines not observed in the earlier work. It is the purpose of this paper to describe the infrared arc spectrum of chromium as revealed by this more recent investigation.

\section{RESULTS}

The observations were made with the concave-grating spectrographs of the National Bureau of Standards, according to our usual practice, which has been described frequently in earlier papers. ${ }^{3}$ The grating ruled with 20,000 lines per inch, giving a dispersion of $3.5 \mathrm{~A} / \mathrm{mm}$ in the first order, was used in making the spectrograms out

\footnotetext{
1 Kiess and Meggers, BS Sci. Pap. 16, 58 (1920) S372.

Astrophys. J. 49, 42 (1919).

BS J. Research 9, 309 (1932) RP473.

$139420-35-6$
} 
to $9100 \mathrm{~A}$. Beyond that limit all the spectrograms were made with the 7,500-lines-per-inch grating, dispersion $10.3 \mathrm{~A} / \mathrm{mm}$. In all the exposures, which varied from a few minutes to two hours, the light source was an arc between lumps of very pure chromium metal between which a direct current of 6 or 7 amperes was maintained under a potential of 220 volts. For each region photographed the appropriate sensitive plate, as recommended by Mees, ${ }^{4}$ was employed after it had been hypersensitized in an ammonia bath. ${ }^{5}$

TABLE 1.-Infrared wave lengths of $\mathrm{Cr} \mathrm{I}$

\begin{tabular}{|c|c|c|c|c|c|c|c|}
\hline$\lambda_{\mathrm{Bir}}$ & $\begin{array}{c}\text { Inten- } \\
\text { sity }\end{array}$ & $\begin{array}{l}\text { Wave num- } \\
\text { ber (vac.) }\end{array}$ & $\begin{array}{l}\text { Term combi- } \\
\text { nation }\end{array}$ & $\lambda_{\mathrm{B} \text { ir }}$ & $\begin{array}{c}\text { Inten- } \\
\text { sity }\end{array}$ & $\begin{array}{l}\text { Wave num- } \\
\text { ber (vac.) }\end{array}$ & $\begin{array}{l}\text { Term combi- } \\
\text { nation }\end{array}$ \\
\hline 11610.48 & 15 & 8610.55 & $z^{5} \mathrm{P}_{3}^{\circ}-c^{5} \mathrm{D}_{4}$ & 9934.52 & 2 & 10063.16 & $y^{7} \mathrm{P}_{3}^{\circ}-e^{5} \mathrm{~S}_{2}$ \\
\hline 11484.50 & 15 & 8705.01 & $z^{5} \mathrm{P}_{2}^{\circ}-c^{5} \mathrm{D}_{3}$ & 9904.47 & 8 & 10093.69 & $a^{3} \mathrm{D}_{3}-z^{3} \mathrm{D}_{2}^{\circ}$ \\
\hline 11472.93 & 10 & 8713.79 & $z^{5} \mathrm{P}_{3}^{\circ}-c^{5} \mathrm{D}_{3}$ & 9900.87 & 15 & 10097.36 & $a^{3} \mathrm{P}_{2}-z^{3} \overline{\mathrm{P}}_{2}^{\circ}$ \\
\hline 11397.96 & 12 & 8771.10 & $z^{5} \mathrm{P}_{1}-c^{5} \mathrm{D}_{2}$ & 9845.19 & 1 & 10154.46 & $y^{7} \mathrm{P}_{2}^{\circ}-e^{5} \mathrm{~S}_{2}$ \\
\hline 11390.63 & 15 & 8776.74 & $z^{5} \mathrm{P}_{2}^{\circ}-c^{5} \mathrm{D}_{2}$ & 9773.30 & 10 & 10229.15 & $a^{3} \mathrm{D}_{2}-z^{3} \mathrm{D}_{3}^{\circ}$ \\
\hline 11379.26 & 5 & 8785.52 & $z^{5} \mathrm{P}_{3}^{\circ}-c^{5} \mathrm{D}_{2}$ & 9758.71 & 2 & 10244.45 & \\
\hline 11339.16 & 15 & 8816.58 & $z^{5} \mathrm{P}_{1}-c^{5} \mathrm{D}_{1}$ & 9752.84 & 4 & 10250.62 & $a^{3} \mathrm{P}_{1}-z^{3} \mathrm{P}_{0}^{\circ}$ \\
\hline 11331.88 & 10 & 8822.25 & $z^{5} \mathrm{P}_{2}^{\circ}-c^{5} \mathrm{D}_{1}$ & 9734.52 & 50 & 10269.91 & $a^{5} \mathrm{G}_{2}-z^{5} \mathrm{~F}_{1}^{0}$ \\
\hline 11310.69 & 12 & 8838.78 & $z^{5} \mathrm{P}_{1}^{\circ}-c^{5} \mathrm{D}_{0}$ & 9730.32 & 25 & 10274.34 & $a^{3} \mathrm{D}_{3}-z^{3} \mathrm{D}_{3}^{\circ}$ \\
\hline 11157.03 & 25 & 8960.51 & $y^{7} \mathrm{P}_{4}^{\circ}-e^{7} \mathrm{~S}_{3}$ & 9670.48 & 50 & 10337.91 & $a^{5} \mathrm{G}_{3}-z^{5} \mathrm{~F}_{2}^{\circ}$ \\
\hline 11044.64 & 5 & 9051.69 & $b^{5} \mathrm{D}_{1}-z^{5} \mathrm{D}_{0}^{\circ}$ & 9667.20 & 25 & 10341.42 & $a^{5} \mathrm{G}_{2}-z^{5} \mathrm{~F}_{2}^{\circ}$ \\
\hline 11015.63 & 30 & 9075.53 & $y^{7} \mathbf{P}_{3}^{\circ}-e^{7} \mathrm{~S}_{3}$ & 9626.30 & 4 & 10385.36 & $a^{3} \mathrm{P}_{1}-z^{3} \mathrm{P}_{1}^{2}$ \\
\hline 10957.19 & 12 & 9123.93 & $b^{5} \mathrm{D}_{2}-z^{5} \mathrm{D}_{1}^{\circ}$ & 9574.25 & 50 & 10441.82 & $a^{5} \mathrm{G}_{4}-z^{5} \mathrm{~F}_{3}^{\circ}$ \\
\hline 10929.90 & 10 & 9146.71 & $b^{5} \mathrm{D}_{0}-z^{5} \mathrm{D}_{1}^{\circ}$ & 9571.76 & 25 & 10444.54 & $a^{5} G_{3}-z^{5} \mathrm{~F}_{3}^{\circ}$ \\
\hline 10905.83 & 25 & 9166.90 & $y^{7} \mathbf{P}_{2}^{\circ}-e^{7} \mathbf{S}_{3}$ & 9568.58 & 4 & 10448.01 & $a^{5} \mathrm{G}_{2}-z^{5} \mathrm{~F}_{3}^{\circ}$ \\
\hline 109 & 2 & 916 & & 9520.13 & 4 & 10501.18 & \\
\hline 10821.62 & 12 & 9238.23 & $b^{5} \mathrm{D}_{3}-z^{5} \mathrm{D}_{2}^{\circ}$ & 9447.00 & 50 & 10582.47 & $\left\{\begin{array}{l}a^{5} \mathrm{G}_{5}-z^{5} \mathrm{~F}_{4}^{\circ} \\
a^{5} \mathrm{G}_{4}-z^{5} \mathrm{~F}^{\circ}\end{array}\right.$ \\
\hline 10816.91 & 8 & 9242.26 & $b^{5} \mathrm{D}_{2}-z^{5} \overline{\mathrm{D}}_{2}^{\circ}$ & & 5 & 10585.43 & $a^{5} \mathrm{G}_{3}-z^{5} \mathrm{~F}_{4}^{\circ}$ \\
\hline 10801.37 & 12 & 9255.55 & $b^{5} \mathrm{D}_{1}-z^{5} \mathrm{D}_{2}^{\circ}$ & .36 & 3 & 10624.45 & \\
\hline 10672.17 & 18 & 9367.60 & $b^{5} \mathrm{D}_{3}-z^{5} \mathrm{D}_{3}^{0}$ & $\begin{array}{l}9409.78 \\
9398.13\end{array}$ & $\begin{array}{l}3 \\
1\end{array}$ & 10637.50 & \\
\hline 10667.53 & 15 & 9371.68 & $b^{5} \mathrm{D}_{2}-z^{5} \mathrm{D}_{3}^{\circ}$ & 9362.06 & 10 & 10678.48 & $a^{3} \mathrm{P}_{1}-z^{3} \mathrm{P}_{2}^{\circ}$ \\
\hline 10647.66 & 12 & 9389.17 & $b^{5} \mathrm{D}_{4}-z^{5} \mathrm{D}_{3}^{\circ}$ & 9313.55 & 8 & 10734.10 & $a^{3} P_{0}-z^{3} P_{1}^{0}$ \\
\hline 10631.42 & 2 & 9403.50 & $u^{5} \mathbf{F}_{5}^{\circ}-e^{5} \mathbf{G}_{6}$ & & 20 & 1075 & $\left\{a^{5} \mathrm{G}_{5}-z^{5} \mathrm{~F}_{5}^{\circ}\right.$ \\
\hline 10550.12 & 3 & 9475.97 & $b^{5} \mathrm{D}_{1}-z^{3} \mathrm{P}_{0}^{\circ}$ & 17 & 20 & 10 & $\left\{a^{5} \mathrm{G}_{4}-z^{5} \mathrm{~F}_{5}^{\circ}\right.$ \\
\hline 10509.96 & 10 & 9512.18 & $b^{5} \mathrm{D}_{3}-z^{5} \mathrm{D}_{4}^{\circ}$ & $\begin{array}{l}9290.44 \\
9263.97\end{array}$ & $\begin{array}{l}50 \\
20\end{array}$ & $\begin{array}{l}10760.80 \\
10791.55\end{array}$ & $\begin{array}{l}a^{5} \mathrm{G}_{6}-z^{5} \mathrm{~F}_{5}^{\circ} \\
a^{3} \mathrm{~F}_{3}-z^{3} \mathrm{G}_{3}^{\circ}\end{array}$ \\
\hline 10486.24 & 20 & 9533.70 & $b^{5} \mathrm{D}_{4}-z^{5} \mathrm{D}_{4}^{\circ}$ & 9208.29 & 25 & 10856.80 & $a^{3} F_{4}-$ \\
\hline $\begin{array}{l}10416.75 \\
1021706\end{array}$ & 2 & 9597.30 & $b^{5} \mathrm{D}_{2}-z^{3} \mathrm{P}_{1}^{0}$ & $\begin{array}{l}9208.29 \\
9148.45\end{array}$ & 6 & 10927.82 & $a^{3} \mathrm{~F}_{3}-z^{3} \mathrm{G}_{4}$ \\
\hline 10217.06 & 1 & 9784.87 & $c^{5} \mathrm{D}_{3}-x^{5} \mathrm{~F}_{4}^{\circ}$ & 9142.60 & 8 & 10934.81 & $x^{3} \mathrm{G}_{5}^{\circ}-e^{3} \mathrm{G}_{5}$ \\
\hline 10197.05 & 3 & 9804.07 & $a^{3} \mathrm{P}_{2}-z^{3} \mathrm{P}_{1}^{\circ}$ & 9141.12 & 7 & 10936.58 & $x^{3} \mathrm{G}_{3}-e^{3} \mathrm{G}_{3}$ \\
\hline 101 & 1 & 9886.63 & $b^{5} \mathrm{D}_{3}-z^{3} \mathrm{P}_{2}^{0}$ & 9140.51 & 8 & 10937.31 & $x^{3} \mathrm{G}_{4}-e^{3} \mathrm{G}_{4}$ \\
\hline 10089.61 & 2 & 9908.48 & $c^{5} \mathrm{D}_{4}-x^{5} \mathrm{~F}_{5}^{\circ}$ & 9128.10 & 2 & 10952.18 & \\
\hline 10083.17 & 5 & 9914.80 & $a^{3} \mathrm{D}_{2}-z^{3} \mathrm{D}_{1}^{\circ}$ & 9113.08 & 2 & 10970.23 & $c^{5} \mathrm{D}_{4}-{ }^{\prime} \mathrm{D}_{3}^{\circ}$ \\
\hline 10080.32 & 15 & 9917.60 & $a^{3} \mathrm{D}_{1}-z^{3} \mathrm{D}_{1}^{0}$ & 9093.76 & 2 & 10993.54 & \\
\hline 9949.06 & 20 & 10048.45 & $a^{3} \mathrm{D}_{2}-z^{3} \mathrm{D}_{2}^{\circ}$ & 9068.30 & 2 & 11024.40 & $c^{5} \mathrm{D}_{4}-x^{\prime}{ }^{5} \mathrm{D}_{4}^{\circ}$ \\
\hline 9946.30 & 7 & 10051.24 & $a^{3} \mathrm{D}_{1}-z^{3} \mathrm{D}_{2}^{\circ}$ & 9059.74 & 5 & 11034.82 & $a^{3} \mathrm{~F}_{4}-z^{3} \mathrm{G}_{5}^{\circ}$ \\
\hline
\end{tabular}

J. Opt. Soc. Am., 25, 80 (1935).

$\checkmark$ Burka, J. Franklin Inst., 189, 25 (1920). 
TABLE 1.-Infrared wave lengths of $\mathrm{Cr} \mathrm{I}$-Continued

\begin{tabular}{|c|c|c|c|c|c|c|c|}
\hline$\lambda_{\mathrm{air}}$ & $\begin{array}{c}\text { Inten- } \\
\text { sity }\end{array}$ & $\begin{array}{c}\text { Wave num- } \\
\text { ber (vac.) }\end{array}$ & $\begin{array}{c}\text { Term combi- } \\
\text { nation }\end{array}$ & $\lambda_{\mathrm{air}}$ & $\begin{array}{c}\text { Inten- } \\
\text { sity }\end{array}$ & $\begin{array}{c}\text { Wave num- } \\
\text { ber (vac.) }\end{array}$ & $\begin{array}{l}\text { Term combi- } \\
\text { nation }\end{array}$ \\
\hline 9035.86 & 20 & 11063.99 & $a^{3} \mathrm{G}_{3}-z^{3} \mathrm{G}_{3}^{\circ}$ & 8530.40 & 1 & 11719.56 & $z^{5} \mathrm{H}_{5}^{\circ}-e^{5} \mathrm{G}_{5}$ \\
\hline 69 & 50 & 11081.36 & $z^{5} \mathrm{P}_{1}^{\circ}-e^{5} \mathrm{~S}_{2}$ & 8511.02 & 7 & 11746.25 & $z^{5} \mathrm{H}_{4}^{0}-e^{5} \mathrm{G}_{3}$ \\
\hline 9017.10 & 75 & 11087.00 & $z^{5} \mathrm{P}_{2}^{0}-e^{5} \mathrm{~S}_{2}$ & 8483.36 & 4 & 11784.55 & $z^{5} \mathrm{H}_{3}^{\circ}-e^{5} \mathrm{G}_{2}$ \\
\hline 9009.95 & 100 & \begin{tabular}{|l|}
11095.80 \\
\end{tabular} & $z^{5} \mathrm{P}_{3}^{\circ}-e^{5} \mathrm{~S}_{2}^{2}$ & 8458.11 & 3 & 11819.73 & \\
\hline 8989.19 & & 11121.42 & & 8455.24 & 12 & 11823.74 & $a^{5} \mathrm{P}_{2}-z^{5} \mathrm{D}_{3}^{\circ}$ \\
\hline 8976.88 & 25 & 11136.67 & $a^{3} \mathrm{G}_{4}-z^{3} \mathrm{G}_{4}^{2}$ & 8450.26 & 15 & 11830.71 & $a^{5} \mathrm{P}_{3}-z^{5} \mathrm{D}_{3}^{0}$ \\
\hline & 2 & 11160.18 & $a^{5} \mathrm{~F}_{2}-z^{5} \mathrm{G}_{2}^{\circ}$ & 8442.95 & 2 & 11841.09 & \\
\hline & 6 & 11162.97 & $a^{5} \mathrm{~F}_{1}-z^{5} \mathrm{G}_{2}^{\circ}$ & 8418.34 & 2 & 11875.57 & \\
\hline $\begin{array}{l}89 \\
80\end{array}$ & 3 & 11170.66 & & $\begin{array}{l}8416.58 \\
8397.04\end{array}$ & 2 & 11878.05 & $a^{5} \mathrm{P}_{1}-q^{5}$ \\
\hline 8947.20 & 35 & 11173.62 & $a^{3} \mathrm{G}_{5}-z^{3} \mathrm{G}_{5}^{\circ}$ & 8397.04 & 6 & 11905.69 & $a^{5} \mathrm{P}_{1}-z^{3} \mathrm{P}_{0}^{0}$ \\
\hline 8943.71 & 2 & 11177.99 & & 8378.52 & 10 & 11932.01 & $z^{3} \mathrm{~F}_{4}^{\circ}-e^{3} \mathrm{G}_{5}$ \\
\hline & 8 & 11183.62 & $a^{5} \mathrm{~F}_{2}-z^{5} \mathrm{G}_{3}^{0}$ & .28 & 20 & 11975.23 & $a^{5} \mathrm{P}_{3}-z^{5} \mathrm{D}_{4}^{0}$ \\
\hline 89 & 3 & 11187.02 & $a^{5} F_{4}-z^{5} \mathrm{G}_{4}^{\circ}$ & 8338.83 & 5 & 11988.80 & $c^{5} \mathrm{D}_{0}-v^{5} \mathrm{~F}_{\mathrm{i}}^{\circ}$ \\
\hline 8929.45 & 2 & 11195.82 & $a^{5} \mathrm{~F}_{5}-z^{5} \mathrm{G}_{5}^{0}$ & 8336.81 & 8 & 11991.70 & $z^{3} \mathrm{~F}_{3}^{\circ}-e^{3} \mathrm{G}_{4}$ \\
\hline 8925.75 & 10 & 11200.48 & $\left\{\begin{array}{l}a^{5} \mathrm{~F}_{3}-z^{5} \mathrm{G}_{4}^{\circ} \\
a^{3} \mathrm{G}_{3}-z^{3} \mathrm{G}_{4}^{\circ}\end{array}\right.$ & 83 & 5 & 12010.96 & $c^{5} \mathrm{D}_{1}-v^{5} \mathrm{~F}_{1}^{\circ}$ \\
\hline & & & & 8322.99 & 3 & 12011.62 & $a^{3} \mathrm{H}_{6}-z^{3} \mathrm{G}_{5}^{\circ}$ \\
\hline 8917.14 & 8 & 11211.28 & $a^{5} \mathrm{~F}_{4}-z^{5} \mathrm{G}_{5}^{\circ}$ & & 20 & & $c^{5} \mathrm{D}_{1}-v^{5} \mathrm{~F}_{2}^{\circ}$ \\
\hline & 15 & 11212.46 & $a^{5} F_{5}-z^{5} \mathrm{G}_{6}^{0}$ & 8318.25 & 8 & 12018.46 & $z^{3} \mathrm{~F}_{2}^{\circ}-e^{3} \mathrm{G}_{3}^{\circ}$ \\
\hline 8898.60 & 1 & 11234.64 & & 8303.19 & \begin{tabular}{r|r}
6 \\
2
\end{tabular} & 12040.26 & $a^{5} \mathrm{P}_{1}-z^{3} \mathrm{P}_{1}^{\circ}$ \\
\hline & 2 & $\begin{array}{l}11270.42 \\
1131466\end{array}$ & $\begin{array}{l}a^{5} \mathrm{~F}_{4}-x^{5} \mathrm{D}_{3} \\
a^{3} \mathrm{G}_{4}-z^{3} \mathrm{G}^{0}\end{array}$ & 8297.58 & 3 & 12048.40 & \\
\hline & & 1131 & & 8296.90 & 4 & 12049.39 & $a^{5} \mathrm{P}_{2}-z^{3} \mathrm{P}_{1}^{\circ}$ \\
\hline 8808.84 & 2 & 11349.12 & $c^{5} \mathrm{D}_{1}-u^{5} \mathrm{P}_{2}^{\circ}$ & $\begin{array}{l}8290.62 \\
8287.38\end{array}$ & $\begin{array}{r}10 \\
25\end{array}$ & 12058.52 & $c^{5} \mathrm{D}_{2}-v^{5} \mathrm{~F}_{2}^{\circ}$ \\
\hline & 2 & 113 & & $\begin{array}{l}8287.38 \\
8286.2\end{array}$ & 25 & 12063.23 & $c^{5} \mathrm{D}_{2}-v^{5} \mathrm{~F}_{3}^{\circ}$ \\
\hline & 1 & 11374.04 & $b^{3} \mathrm{P}_{2}-z^{3} \mathrm{D}_{\mathrm{i}}^{\circ}$ & $\begin{array}{l}8286.33 \\
8285.72\end{array}$ & 2 & $\begin{array}{l}12064.76 \\
12065.64\end{array}$ & \\
\hline $\begin{array}{l}8.31 \\
6.96\end{array}$ & $\begin{array}{l}1 \\
4\end{array}$ & $\begin{array}{l}11375.63 \\
11377.37\end{array}$ & $c^{5} \mathrm{D}_{3}-u^{5} \mathrm{P}_{3}^{\circ}$ & & & & \\
\hline & & & 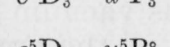 & 8273.80 & 3 & 12083.03 & $x^{5} \mathrm{~F}_{5}^{\circ}-e^{5} \mathrm{G}_{6}$ \\
\hline 8784.15 & $\begin{array}{l}2 \\
5\end{array}$ & $\begin{array}{l}11381.02 \\
11304.75\end{array}$ & $c^{5} \mathrm{D}_{0}-u^{5} \mathrm{P}_{1}$ & $\begin{array}{l}8273.20 \\
8264.27\end{array}$ & $\begin{array}{r}3 \\
2\end{array}$ & $\begin{array}{l}12083.90 \\
12096.96\end{array}$ & $x^{5} \mathrm{~F}_{\mathrm{i}}^{\circ}-e^{5} \mathrm{G}_{5}$ \\
\hline $\begin{array}{c}.56 \\
.12\end{array}$ & $\begin{array}{l}5 \\
4\end{array}$ & $\begin{array}{l}1132 \\
1140\end{array}$ & $\begin{array}{l}c^{0} \mathrm{D}_{2}-u^{5} \mathrm{P}_{2} \\
c^{5} \mathrm{D}_{1}-u^{5} \mathrm{P}_{1}\end{array}$ & 8261.95 & 8 & 12100.36 & $a^{3} \mathrm{H}_{4}-z^{3} \mathrm{G}_{4}$ \\
\hline 8753.50 & 5 & 11420.87 & $b^{3} \mathrm{P}_{1}-z^{3} \mathrm{D}_{1}^{0}$ & 8240.67 & 2 & 12131.61 & $c^{5} \mathrm{D}_{0}-w^{5} \mathrm{D}_{1}^{\circ}$ \\
\hline 874 & 2 & 11433.86 & $b^{3} \mathrm{P}_{0}-z^{3} \mathrm{D}_{1}^{\circ}$ & & 12 & & \\
\hline 8732.17 & 3 & .77 & $c^{5} \mathrm{D}_{2}-u^{5} \mathrm{P}_{1}^{\circ}$ & 8235.89 & 30 & 12138.65 & $\begin{array}{l}c^{0} \mathrm{D}_{3}-v^{5} \mathrm{~F} \\
c^{5} \mathrm{D}_{3}-v^{5} \mathrm{~F}\end{array}$ \\
\hline & | & 1146 & $c^{5} \mathrm{D}_{3}-u^{5} \mathrm{P}_{2}^{1}$ & 8225.67 & 3 & 12153.73 & $c^{5} \mathrm{D}_{1}-w^{5} \mathrm{D}_{\mathrm{i}}$ \\
\hline 8716.09 & 1 & 11469.89 & & 8224.09 & 8 & 12156.06 & $a^{3} \mathrm{H}_{5}-z^{3} \mathrm{G}_{5}^{\circ}$ \\
\hline & 12 & 1148 & $a^{5} \mathrm{P}_{1}-z^{5} \mathrm{D}_{0}^{\circ}$ & 8218.07 & 3 & 12164.97 & \\
\hline & & & $c$ & & 5 & 12167.62 & $c^{5} \mathrm{D}_{1}-w^{5} \mathrm{D}_{2}^{\circ}$ \\
\hline 8687.47 & 7 & 115 & $b^{3} \mathrm{P}_{2}-z^{3} \mathrm{D}_{2}^{\circ}$ & & 4 & 12199.40 & $c^{5} \mathrm{D}_{2}-w^{5} \mathrm{D}_{1}^{\circ}$ \\
\hline & 3 & & $a^{5} \mathrm{~F}_{5}-x^{5} \mathrm{I}$ & 8188.77 & 3 & 12208.50 & \\
\hline & t & 11522.89 & & 69 & 5 & 12213.09 & $c^{5} \mathrm{D}_{2}-w^{5} \mathrm{D}_{2}^{\circ}$ \\
\hline 57 & 1 & 11552.76 & & 8169.80 & 5 & 12236.84 & $c^{5} \mathrm{D}_{0}-u^{5} \mathrm{~F}_{\mathrm{i}}^{\mathrm{i}}$ \\
\hline & 3 & 4.53 & $b^{3} \mathrm{P}_{1}-z^{3} \mathrm{D}_{2}^{\circ}$ & 8167.94 & 4 & 12239.63 & \\
\hline 8643.03 & 12 & 115 & $a^{5} \mathrm{P}_{1}-z^{5} \mathrm{D}_{1}^{\circ}$ & 816 & 7 & 1.55 & $\left\{c^{5} \mathrm{D}_{4}-v^{5} \mathrm{~F}_{4}^{\circ}\right.$ \\
\hline $\begin{array}{l}.26 \\
.26\end{array}$ & $\begin{array}{r}10 \\
2\end{array}$ & $\begin{array}{l}115 \\
116\end{array}$ & & & 35 & & $c^{5} \mathrm{D}$ \\
\hline 8583.01 & 10 & 11647.73 & $z^{5} \mathrm{H}_{7}^{\circ}-e^{5}$ & 81 & 6 & 12259.05 & $c^{5} \mathrm{D}_{1}-u^{5} \mathrm{~F}_{\mathrm{i}}^{\circ}$ \\
\hline 8562.56 & & 11675.55 & $z^{5} \mathrm{H}_{6}^{0}-e^{5} \mathrm{G}_{5}$ & 8128.28 & 8 & 12299.35 & $c^{5} \mathrm{D}_{1}-u^{5} \mathrm{~F}_{2}^{\circ}$ \\
\hline 855 & 5 & 116 & $\left.a^{5}\right]$ & 81 & 2 & 12304.57 & $c^{5} \mathrm{I}$ \\
\hline & & & & & 3 & 12313.21 & $c^{5}$ \\
\hline 854 & 12 & 11694.30 & $a^{5} \mathrm{P}_{2}-z^{5} \mathrm{D}_{2}^{\circ}$ & 8098.24 & 5 & 12344.97 & $c^{5} \mathrm{D}_{2}-u^{5} \mathrm{~F}_{2}^{\circ}$ \\
\hline & 10 & 11701.29 & $a^{5} \mathrm{P}_{3}-z^{5} \mathrm{D}_{2}^{0}$ & 8084.98 & 10 & 12365.22 & $c^{5} \mathrm{D}_{3}-w^{5} \mathrm{D}_{4}$ \\
\hline 8537.80 & 8 & 11709.41 & $z^{5} \mathrm{H}_{5}^{\circ}-e^{5} \mathrm{G}_{4}$ & 8061.27 & $|10|$ & 12401.59 & $c^{5} \mathrm{D}_{2}-u^{5} \mathrm{~F}_{3}^{\circ}$ \\
\hline
\end{tabular}


TABLE 1.-Infrared wave lengths of $\mathrm{Cr} \mathrm{I}-$ Continued

\begin{tabular}{|c|c|c|c|c|c|c|c|}
\hline$\lambda_{\mathrm{air}}$ & \begin{tabular}{|} 
Inten- \\
sity
\end{tabular} & $\begin{array}{l}\text { Wave num- } \\
\text { ber (vac.) }\end{array}$ & $\begin{array}{l}\text { Term combi- } \\
\text { nation }\end{array}$ & $\lambda_{\mathrm{B} \text { ir }}$ & $\begin{array}{c}\text { Inten- } \\
\text { sity }\end{array}$ & $\begin{array}{l}\text { Wave num- } \\
\text { ber (vac.) }\end{array}$ & $\begin{array}{l}\text { Term combi- } \\
\text { nation }\end{array}$ \\
\hline 8050.68 & 2 & 12417.92 & & 7910.50 & 10 & 12637.95 & $z^{3} \mathrm{H}_{5}^{\circ}-e^{3} \mathrm{G}_{4}$ \\
\hline 8045.33 & 7 & 12426.16 & & 7908.30 & 12 & 12641.47 & $z^{3} \mathrm{H}_{6}^{0}-e^{3} \mathrm{G}$ \\
\hline 8018.04 & 3 & 12468.45 & $c^{5} \mathrm{D}_{4}-w^{5} \mathrm{D}_{4}^{\circ}$ & 7885.00 & 4 & 12678.82 & $c^{5} \mathrm{D}_{2}-u^{\prime}{ }^{5} \mathrm{~F}_{3}^{\circ}$ \\
\hline 8014.88 & 5 & 12473.36 & $c^{5} \mathrm{D}_{3}-u^{5} \mathrm{~F}_{3}^{\circ}$ & 7771.74 & 8 & 12863.59 & \\
\hline 799 & 10 & 12511.42 & & 7763.00 & 4 & 12878.08 & \\
\hline 7989.36 & 12 & 12513.21 & $c^{5} \mathrm{D}_{3}-u^{5} \mathrm{~F}_{4}^{\circ}$ & 7749.25 & 1 & 12900.93 & \\
\hline 707121 & 4 & 12541.54 & $c^{5} \mathrm{D}_{3}^{0}-u^{\prime}{ }^{5} \mathrm{~F}_{4}^{0}$ & 7734.00 & 3 & 12926.37 & $b^{3} \mathrm{~F}_{2}-z^{3} \mathrm{~F}$ \\
\hline 7942.02 & 20 & 12587.80 & $c^{5} \mathrm{D}_{4}^{0}-u^{5} \mathrm{~F}_{5}^{0}$ & 7726.02 & 3 & 12939.72 & $b^{3} \mathrm{~F}_{3}-z^{3} \mathrm{~F}_{2}$ \\
\hline 7924 & 3 & 12616.33 & $c^{5} \mathrm{D}_{4}-u^{5} \mathrm{~F}_{4}^{\circ}$ & 7724.50 & 1 & 12942.27 & \\
\hline & 10 & 12626.23 & $z^{3} \mathrm{H}_{4}^{2}-e^{3} \mathrm{G}_{3}^{2}$ & 7722.89 & 5 & 12944.96 & $b^{3} \mathrm{~F}_{4}-z^{3} \mathrm{~F}_{4}^{\circ}$ \\
\hline
\end{tabular}

The new wave lengths, entered in table 1, are the means of from: 2 to 12 observations, including those given in the earlier paper. These are referred to the iron standards,${ }^{6}$ which were observed in the secondand third-order spectra in juxtaposition with the first-order chromium spectrum. Following each recorded wave length is an estimated intensity, the wave number in vacuum, and the classification of the line.

The term combinations assigned to the classified lines are based on previously known terms of CrI and on the new and hitherto unpublished terms given in tables 2 and 3 . The term structure of $\mathrm{Cr}$ I is built up, theoretically, of septet, quintet, triplet, and singlet systems. Of these, the septets and quintets account for nearly all the prominent lines of the spectrum, which according to King ${ }^{7}$ are excited at the lowest temperature stages of his vacuum furnace. Except for three odd terms of the triplet system, all the previously known terms of Cr I belong to the septet and quintet systems. They are given in an earlier paper of this Journal ${ }^{8}$ and also by Catalan and Sancho, ${ }^{9}$ who, in addition, give a list of classified lines derived from them. The new terms of tables 2 and 3 belong mostly to the triplet system.

TABLE 2.-Even terms of $\mathrm{Cr} \mathrm{I}$

\begin{tabular}{|c|c|c|c|c|c|}
\hline Term & $\nu$ & $\Delta \nu$ & Term & $\nu$ & $\Delta \nu$ \\
\hline $\begin{array}{l}c^{5} \mathrm{D}_{4-\ldots} \\
c^{5} \mathrm{D}_{3-\ldots} \\
c^{5} \mathrm{D}_{2-\ldots} \\
c^{5} \mathrm{D}_{1-\ldots} \\
c^{5} \mathrm{D}_{0 \ldots}\end{array}$ & $\begin{array}{l}35397.7 \\
35501.0 \\
35572.8 \\
35618.4 \\
35640.6\end{array}$ & $\begin{array}{r}-103.3 \\
-71.8 \\
-45.6 \\
-22.2\end{array}$ & $\begin{array}{l}a^{5} \mathrm{~F}_{5--} \\
a^{5} \mathrm{~F}_{4---} \\
a^{5} \mathrm{~F}_{3--} \\
a^{5} \mathrm{~F}_{2---} \\
a^{5} \mathrm{~F}_{1---}\end{array}$ & $\begin{array}{l}31393.1 \\
31377.8 \\
31364.1 \\
31355.0 \\
31352.3\end{array}$ & $\begin{array}{r}15.3 \\
13.7 \\
9.1 \\
2.7\end{array}$ \\
\hline $\begin{array}{l}f^{5} \mathrm{D}_{4} \ldots \ldots \ldots \\
f^{5} \mathrm{D}_{3} \\
f^{5} \mathrm{D}_{2} \\
f^{5} \mathrm{D}_{1} \\
f^{5} \mathrm{D}_{0} \ldots \ldots\end{array}$ & $\begin{array}{l}55209.0 \\
54987.0 \\
54818.7 \\
54671.8\end{array}$ & $\begin{array}{l}222.0 \\
168.3 \\
146.9\end{array}$ & $\begin{array}{l}e^{5} \mathrm{G}_{6} \\
e^{5} \mathrm{G}_{5} \\
e^{5} \mathrm{G}_{4} \\
e^{5} \mathrm{G}_{3 \ldots} \\
e^{5} \mathrm{G}_{2 \ldots}\end{array}$ & $\begin{array}{l}57388.9 \\
57382.7 \\
57372.6 \\
57361.2 \\
57350.6\end{array}$ & $\begin{array}{r}6.2 \\
10.1 \\
11.4 \\
10.6\end{array}$ \\
\hline
\end{tabular}

6 Trans. Int. Astron. Union, 3, 77 (1928).

7 Astrophys. J. 41, 86 (1915).

8 Kiess, BS J. Research 5, 775 (1930) RP229. Note.-The term listed in this paper as $y^{5} \mathrm{D}_{0}^{\circ}=41124.7$ should read 41224.7 .

Anales Soc. Espan. Fis. Quim., 19, 327 (1931). 
Table 2.-Even terms of Cr I-Continued

\begin{tabular}{|c|c|c|c|c|c|}
\hline Term & $\nu$ & $\Delta \nu$ & Term & $\nu$ & $\Delta \nu$ \\
\hline $\begin{array}{l}a^{3} \mathrm{P}_{2} \\
a^{3} \mathrm{P}_{1} \mathrm{P}_{0}\end{array}$ & $\begin{array}{l}24093.2 \\
23511.9 \\
23163.2\end{array}$ & $\begin{array}{l}581.3 \\
348.7\end{array}$ & $\begin{array}{l}a^{3} \mathrm{G}_{5--} \\
a^{3} \mathrm{G}_{4--} \\
a^{3} \mathrm{G}_{3--}\end{array}$ & $\begin{array}{l}25038.3 \\
24897.3 \\
24833.7\end{array}$ & $\begin{array}{r}141.0 \\
63.6\end{array}$ \\
\hline $\begin{array}{l}b^{3} \mathrm{P}_{2} \\
b^{3} \mathrm{P}_{1} \ldots \\
b^{3} \mathrm{P}_{0}\end{array}$ & $\begin{array}{l}27223.0 \\
27176.0 \\
27163.1\end{array}$ & $\begin{array}{l}47.0 \\
12.9\end{array}$ & $\begin{array}{l}b^{3} \mathrm{G}_{5--} \\
b^{3} \mathrm{G}_{4--} \\
b^{3} \mathrm{G}_{3--}\end{array}$ & $\begin{array}{l}27816.7 \\
27703.6 \\
27597.2\end{array}$ & $\begin{array}{l}113.1 \\
106.4\end{array}$ \\
\hline $\begin{array}{l}a^{3} \mathrm{D}_{3} \ldots \\
a^{3} \mathrm{D}_{2 \ldots} \\
a^{3} \mathrm{D}_{1 \ldots}\end{array}$ & $\begin{array}{l}28637.0 \\
28682.2 \\
28679.6\end{array}$ & $\begin{array}{r}-45.2 \\
2.6\end{array}$ & $\begin{array}{l}e^{3} \mathrm{G}_{5} \\
e^{3} \mathrm{G}_{4-} \\
e^{3} \mathrm{G}_{3 \ldots}\end{array}$ & $\begin{array}{l}57990.1 \\
57992.0 \\
57984.9\end{array}$ & $\begin{array}{r}-1.9 \\
7.1\end{array}$ \\
\hline $\begin{array}{l}b^{3} \mathrm{D}_{3} \\
b^{3} \mathrm{D}_{2 \ldots} \\
b^{3} \mathrm{D}_{1}\end{array}$ & $\begin{array}{l}31009.0 \\
31028.4 \\
31048.8\end{array}$ & $\begin{array}{l}-19.4 \\
-20.4\end{array}$ & $\begin{array}{l}a^{3} \mathrm{H}_{6-\ldots} \\
a^{3} \mathrm{H}_{5-} \\
a^{3} \mathrm{H}_{4-}\end{array}$ & $\begin{array}{l}24200.1 \\
24055.9 \\
23933.7\end{array}$ & $\begin{array}{l}144.2 \\
122.2\end{array}$ \\
\hline $\begin{array}{l}a^{3} \mathrm{~F}_{4} \ldots \\
a^{3} \mathrm{~F}_{3-1} \\
a^{3} \mathrm{~F}_{2}\end{array}$ & $\begin{array}{l}25177.2 \\
25106.1 \\
24940.5\end{array}$ & $\begin{array}{r}71.1 \\
165.6\end{array}$ & $\begin{array}{l}a^{3} \mathrm{I}_{7} \ldots \ldots \\
a^{3} \mathrm{I}_{6} \\
a^{3} \mathrm{I}_{5}\end{array}$ & $\begin{array}{l}31047.8 \\
31049.1 \\
31055.2\end{array}$ & $\begin{array}{l}-1.3 \\
-6.1\end{array}$ \\
\hline $\begin{array}{l}b^{3} \mathrm{~F}_{4 \ldots} \\
b^{3} \mathrm{~F}_{3 \ldots} \\
b^{3} \mathrm{~F}_{2 \ldots}\end{array}$ & $\begin{array}{l}33113.2 \\
33060.7 \\
33040.2\end{array}$ & $\begin{array}{l}52.5 \\
20.5\end{array}$ & & & \\
\hline
\end{tabular}

TABle 3.-Odd terms of Cr I

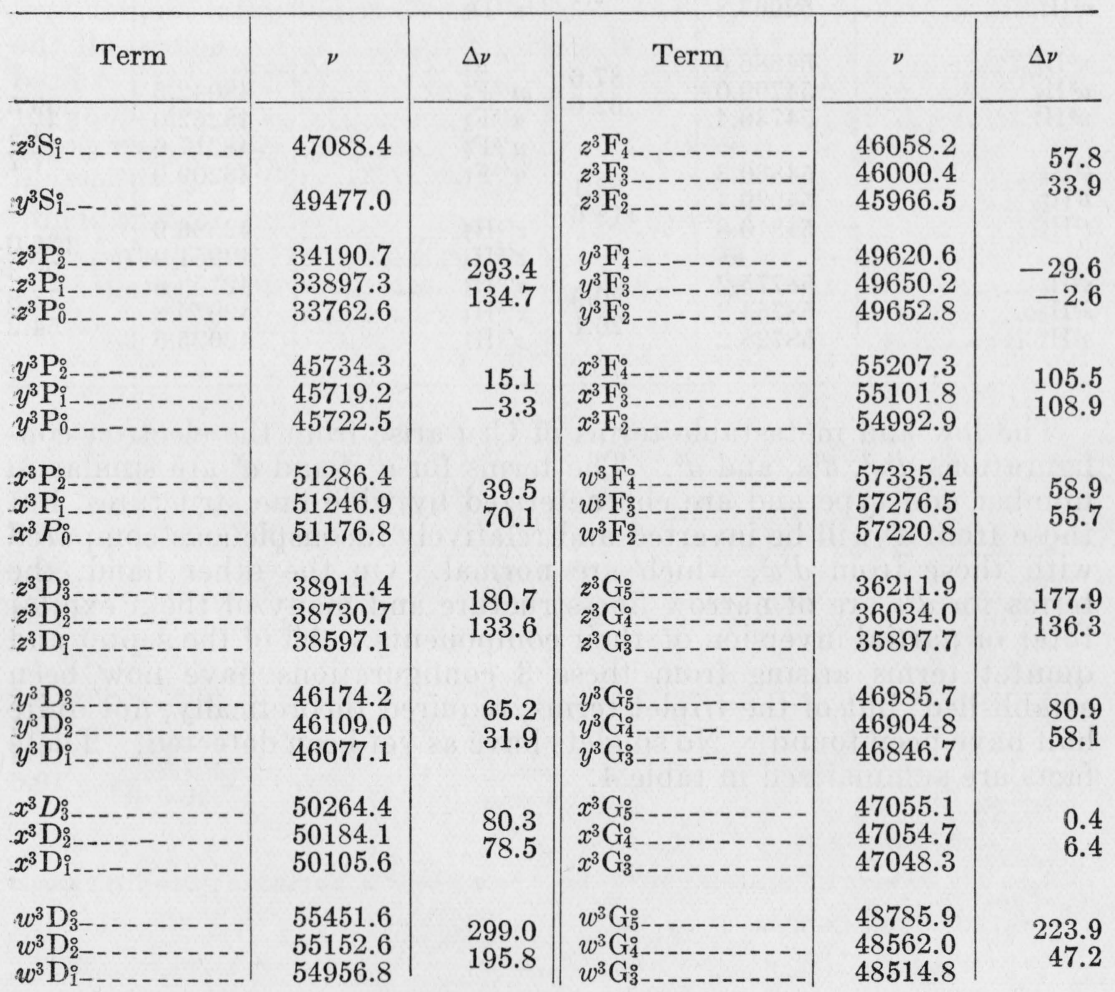


TABLE 3.-Odd terms of Cr I-Contin ued

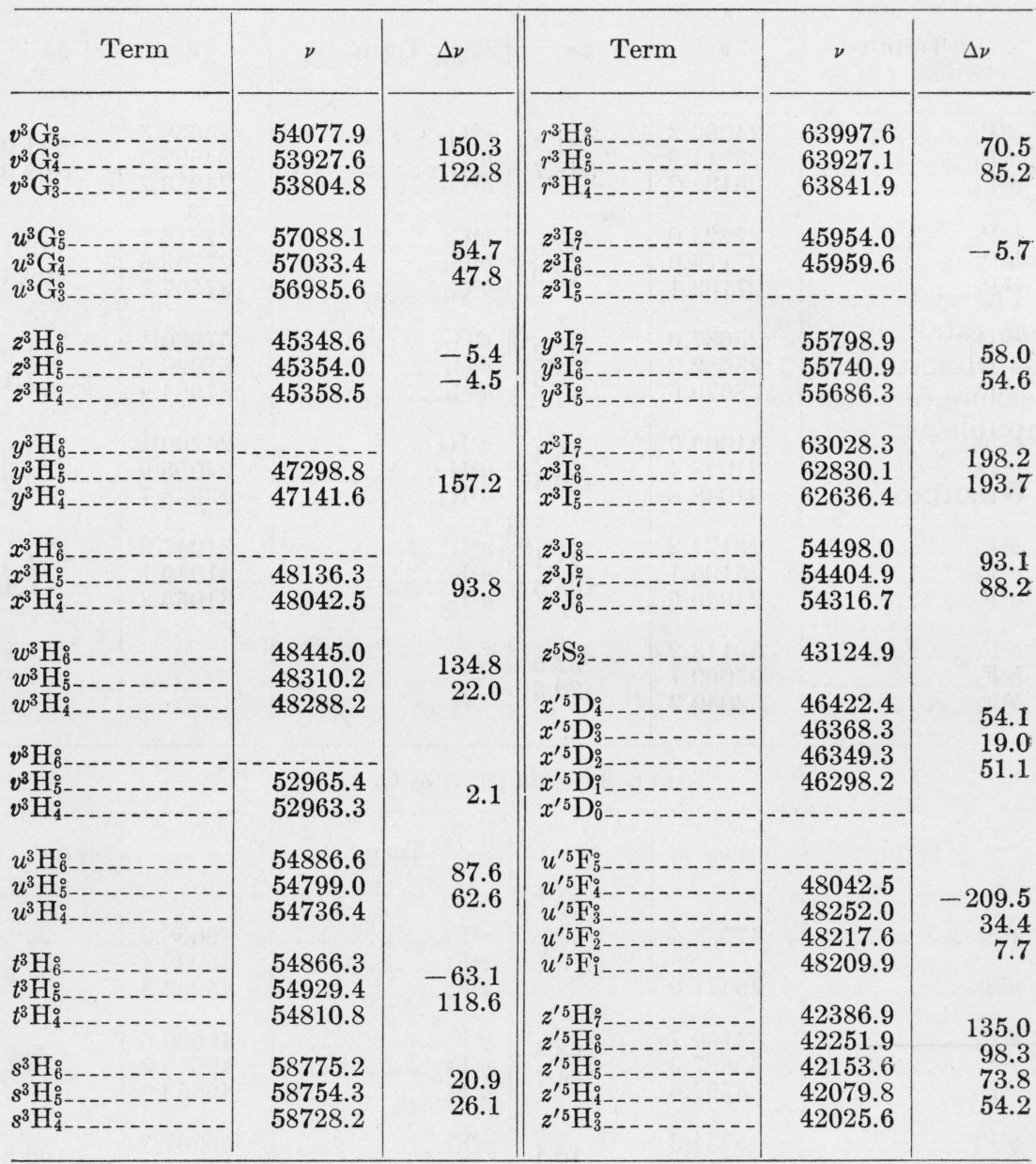

The low and metastable terms of $\mathrm{Cr}$ I arise from the electron configurations $d^{4} s^{2}, d^{5} s$, and $d^{6}$. The terms for $d^{4} s^{2}$ and $d^{6}$ are similar in number and type and are characterized by wide fine structures, but those from $d^{6}$ will be inverted and relatively inconspicuous compared with those from $d^{4} s^{2}$, which are normal. On the other hand, the terms for $d^{5} s$ are of narrow fine-structure and many of them exhibit total or partial inversion of their components. All of the septet and quintet terms arising from these 3 configurations have now been established; but of the triplet terms, required theoretically, not quite half have been found. No singlets have as yet been detected. These facts are summarized in table 4 . 
TABLE 4.-Low and metastable terms of $\mathrm{Cr} \mathrm{I}$

\begin{tabular}{|c|c|}
\hline $\begin{array}{c}\text { Electron } \\
\text { configuration }\end{array}$ & Observed terms \\
\hline $\begin{array}{l}3 d^{4} 4 s^{2} \\
3 d^{5} 4 s \\
3 d^{6}\end{array}$ & $\begin{array}{l}a^{5} \mathrm{D}, a^{3} \mathrm{H}, b^{3} \mathrm{G}, a^{3} \mathrm{~F}, a^{3} \mathrm{P} \\
a^{7} \mathrm{~S}, a^{5} \mathrm{~S}, a^{5} \mathrm{G}, a^{3} \mathrm{G}, a^{5} \mathrm{~F}, b^{3} \mathrm{~F}, b^{5} \mathrm{D}, a^{3} \mathrm{D}, a^{5} \mathrm{P}, b^{3} \mathrm{P}, a^{3} \mathrm{I}, l^{3} \mathrm{D} \\
c^{5} \mathrm{D}\end{array}$ \\
\hline
\end{tabular}

The identity of many of the triplet terms reported in this paper has been established by the excellent Zeeman effect observations made at the Mount Wilson Observatory by Mr. H. D. Babcock. It is a pleasure to acknowledge my indebtedness to him for placing his unpublished work at my disposal.

Washington, April 17, 1935. 